\title{
Controlling soliton excitations in Heisenberg spin chain through magic angle
}

\author{
Jing Lu, ${ }^{1,2}$ Lan Zhou, ${ }^{1}$ Le-Man Kuang, ${ }^{1}$ and C. P. Sun ${ }^{2}$, * \\ ${ }^{1}$ Department of Physics, Hunan Normal University, Changsha 410081, China \\ ${ }^{2}$ Institute of Theoretical Physics, Chinese Academy of Sciences, Beijing, 100080,China
}

\begin{abstract}
We study the nonlinear dynamics of collective excitation in a $N$-site $X X Z$ quantum spin chain, which is manipulated by an oblique magnetic field. We show that, when the tilted field is applied along the magic angle $\theta_{0}= \pm \arccos \sqrt{1 / 3}$, the anisotropic Heisenberg spin chain becomes isotropic and thus an free propagating spin wave is stimulated. And in the regime of the tilted angle larger and smaller then the magic angle, two types of nonlinear excitations appear, which are bright soliton and dark soliton.
\end{abstract}

PACS numbers: 05.45.Yv, 75.10.Pq, 76.60.Lz

\section{INTRODUCTION}

A crucial challenge for quantum information processing (QIP) is perfectly transmitting a quantum state from one place to another as well as storing the information of a quantum state. Recently, as much attentions have been paid to the solid-based quantum computing, there are many proposals for quantum state transfer and storage, using quantum spin systems as quantum data bus and quantum memory [1, 2, 3, 44.

In other hand the Heisenberg chain is a typical spin system in condensed-matter physics. It has attracted considerable attentions for a long time in describing various magnetic properties of materials. As a strong correlated system [5, [6, 7, 8,9$]$, it can display rich nonlinearities induced by inter-spin interaction. The typical nonlinear phenomenon is the solitary wave, that is a wave packet propagate without either energy loss and wave packet spreading. In this sense it is quite natural to consider the role of soliton wave in quantum state transfer and quantum information storage.

Generally there are two major approaches to theoretically study the solitary excitation in the one-dimension (1D) Heisenberg chain 11, 12, 13, 14, 15, 16, 17, 18, 19]. 1) each spin is represented as a Bloch vector, and a solitary excitation is found by investigating the nonlinear dynamics of this classical variable 12, 13]. 2) Another procedure is to employ the boson mappings of spin operators via the Holstein-Primakoff transformation and JordanSchwinger realization[10]. By making use of the spincoherent-state representation, a solvable nonlinear differential equation can be deduced from the Heisenberg equation for the mapping boson operators, therefore a solitary excitation is implied in the system [14, 15, 16, 17, 18, 19].

Our present investigation is motivated by the well known knowledge in nuclear magnetic resonance (NMR) that the effective spin-spin interaction can be controlled by the angle of the external applied magnetic field [10]. Here, to some extent, the inter-spin coupling can be can-

\footnotetext{
*Electronic address:

URL: http://www.itp.ac.cn/ suncp

suncp@itp.ac.cn
}

celed effectively by the magic angle. Nowadays together with the spin echo technology, magic angle manipulation has became a necessary tool for controlling the interacting spin system to be free of decoherence due to the interspin couplings. In this paper, we apply a magnetic field on a N-site XXZ spin chain, which is rotated around the $\mathrm{y}$-axis by an amount of magnitude $\theta$, and study how to manipulate a soliton excitation by this oblique field. It is known that the ferromagnetic order is completely determined by the direction of the external magnetic field. Indeed, our investigation shows that the switch between the bright soliton and the dark soliton is controlled by $\theta$, whose tangent is defined as the ratio between the $\mathrm{z}$ component and the $\mathrm{x}$ component of the magnetic field; and at the turning magnitude referred to as the magic angle $\theta_{0}$, the original anistropic chain becomes isotropic, therefore only an ideal spin wave is stimulated as an effective plane wave. Here, we employ the second approach mentioned above, but go beyond the spin wave approach by considering the nonlinear effect of the collective spin excitation. Generally, the spin wave approach is regarded as a mean field method with a given order parameter. Due to the ignorance of the nonlinear fluctuation, the spin wave approach does not cover the quantum fluctuation in the nonlinear regime. However our approach overcomes this disadvantage.

The remainder of this paper is organized as follows. In section II] we present our model - a Heisenberg spin chain with anisotropic coupling in an oblique magnetic field. The approach we use is in sections [II] and [V] Here the quasi-classical equation of motion is obtained, which is a nonlinear Schrödinger (NLS) equation. In section $\mathrm{V}$, the time evolution of nonlinear excitations is studied. The bright soliton and dark soliton can be excited as $\theta$ shifted to the left or the right of the magic angle. In section VI, we give a remark about the relationship between the soliton wave propagation and perfect quantum state transfer. 


\section{A SPIN CHAIN MODEL}

We consider a one-dimensional XXZ spin chain with $N$ spins in an homogeneous external magnetic field

$$
\mathbf{B}=B\left(e_{x} \sin \theta+e_{z} \cos \theta\right)
$$

Denote the spin operator at the $j$ th site by Pauli spin operator $S_{j}^{x}, S_{j}^{y}$, and $S_{j}^{z}$. Then Hamiltonian $H$ of this system reads

$$
H=-J \sum_{j=1}^{N}\left(\hat{S}_{j}^{x} \hat{S}_{j+1}^{x}+\hat{S}_{j}^{y} \hat{S}_{j+1}^{y}+\Delta \hat{S}_{j}^{z} \hat{S}_{j+1}^{z}\right)+\mathbf{B} \cdot \mathbf{S}
$$

where $\mathbf{S}=\sum_{j} \mathbf{S}_{j}$ is the total spin operator. Three types of interactions are included in Eq.(2): 1) the isotropic part of nearest neighbor exchange interaction $J_{x}=J_{y}=$ $J>0$. 2) the anisotropic part of nearest neighbor exchange interaction, which is characterized by a dimensionless parameter $\Delta=J_{z} / J$. 3) the external homogeneous field $\mathbf{B}$, which is specified by its components $B_{x}=B \sin \theta$ and $B_{z}=B \cos \theta$. Here the magnitude of the magnetic field $B=\sqrt{B_{x}^{2}+B_{z}^{2}}$, and the oblique angle $\theta=\arctan \left(B_{z} / B_{x}\right)$.

Obviously, the last term is proportional to the total spin $z$-component $\hat{S}_{\text {total }}^{z}=\sum_{\mathbf{i}} \hat{S}_{\mathbf{i}}^{z}$, which is conserved and thus has common eigenstates with the total Hamiltonian. Correspondingly, the Hilbert space of the system can be decomposed into a direct sum of numerous subspaces $V(M)$ specified by the total spin number $M$ along z-axis. It had been proved [20, 21] that, on a finite simple cubic lattice, the ground state of the $X X Z$ model is nondegenerate in an subspace $V(M)$. In particular, its global ground state $\Psi_{0}(\Delta)$ is just the ground state of the model in the subspace $V(M=0)$ When the lattice is finite, the ground state energy $E_{0}(\Delta)$ and the spin correlation function $\left\langle\hat{S}_{\mathbf{i}}^{z} \hat{S}_{\mathbf{i}}^{z}\right\rangle$ are analytical with respect to $\Delta$.

Here, we assume that the external magnetic field is much larger than the inter-spin interaction. Therefore we switch to a new "reference frame" with new $Z$-axis along the quantized direction of spin along the effective field $\mathbf{B}$. The corresponding $\mathbf{S}_{j} \rightarrow \mathbf{L}_{j}$ transformation for each spin at site $j$ reads

$$
\begin{aligned}
L_{j}^{x} & =\hat{S}_{\mathbf{j}}^{x} \cos \theta-\hat{S}_{\mathbf{j}}^{z} \sin \theta, \\
L_{j}^{y} & =\hat{S}_{\mathbf{j}}^{y}, \\
L_{j}^{z} & =\hat{S}_{j}^{z} \cos \theta+\hat{S}_{j}^{x} \sin \theta .
\end{aligned}
$$

Therefore Hamiltonian in Eq. (2) is decomposed into a direct sum of the $S O(3)$ irreducible tensors with respect to $\mathbf{L}_{j}$, i.e,

$$
H=B L_{z}+\sum_{M=-2}^{M=2} H_{M}
$$

which is written according to the irreducible representation $D^{[L]}(L=2,1,0)$ of $S O(3)$ group. Here, $L_{z}=\sum_{j} L_{j}^{z}$ is the third component of the total angular momentum. With $\delta=(\Delta-1)$,

$$
\begin{aligned}
H_{0}= & -\left(1+\frac{\delta}{2} \sin ^{2} \theta\right) J \sum_{j} \mathbf{L}_{j} \cdot \mathbf{L}_{j+1} \\
& -\frac{\delta}{2} J\left(3 \cos ^{2} \theta-1\right) \sum_{j} L_{j}^{z} L_{j+1}^{z}
\end{aligned}
$$

belongs to the zero rank representation $D^{[0]}$ of $S O(3)$, while

$$
H_{ \pm 1}=\frac{\delta}{4} J \sin 2 \theta \sum_{j}\left(L_{j}^{z} L_{j+1}^{ \pm}+L_{j}^{ \pm} L_{j+1}^{z}\right)
$$

and

$$
H_{ \pm 2}=-\frac{\delta}{4} J \sin ^{2} \theta \sum_{j} L_{j}^{ \pm} L_{j+1}^{ \pm}
$$

belong to the 1 st and 2 nd rank representation $D^{[1]}$ and $D^{[2]}$ respectively. The $M$ th order tensors $H_{M}$ satisfy

$$
\left[L_{z}, H_{M}\right]=M H_{M},\left[L_{z}, H_{0}\right]=0 .
$$

From the point of view of perturbation theory, the eigenvalues of the Hamiltonian are those of $B L_{z}$ at the 0th order, i.e. $L_{z}=M$, which means the ground state has all spins parallel to the field and it is not degenerate. However, the excited state, where one or two spins are flipped with respect to the ground state, are degenerate. Therefore, when the perturbation theory is applied, we diagonalize Hamiltonian $H$ in each subspace of a given $M$, which means that at lowest order, Hamiltonian $H_{0}$ is kept. Thus, the $H_{0}$ plays an indispensable role to govern the dynamics of the spin chain. It can be found directly from Hamiltonian $H_{0}$ that the spin-spin interaction is controlled by the direction of the applied magnetic field. When $\theta=\theta_{0}= \pm \arccos \sqrt{1 / 3}$, the original anisotropic chain becomes isotropic.

We further explain the above argument from the point view of the representation theory of $\mathrm{SO}(3)$ together with rotating wave approximation. Sandwiched by the common states $\left|J, M^{\prime}\right\rangle$ and $\left|J, M^{\prime \prime}\right\rangle$ of $H_{0}$ and $L_{z}$, the equations (8) lead to

$$
\begin{aligned}
& \left\langle J, M^{\prime}\left|\left[L_{z}, H_{M}\right]\right| J, M^{\prime \prime}\right\rangle \\
= & \left(M^{\prime}-M^{\prime \prime}\right)\left\langle J, M^{\prime}\left|H_{M}\right| J, M^{\prime \prime}\right\rangle \\
= & M\left\langle J, M^{\prime}\left|H_{M}\right| J, M^{\prime \prime}\right\rangle
\end{aligned}
$$

here $M=M^{\prime}-M^{\prime \prime}$ for the nonvanishing matrix element $\left\langle J, M^{\prime}\left|H_{M}\right| J, M^{\prime \prime}\right\rangle$. In the interaction picture, the offdiagonal elements $\left\langle J, M^{\prime}\left|H_{M}\right| J, M^{\prime \prime}\right\rangle$ are fast changing with high frequency, therefore the terms with $|M|=1$ and $|M|=2$ in the total Hamiltonian can be ignored [10].

\section{QUASI-CLASSICAL MOTION EQUATION FOR FERROMAGNETIC SPIN CHAIN}

With the above considerations, the $H_{M}(M= \pm 1, \pm 2)$ are the first and second order tensor operators that trans- 
form according to the representation $D^{[j=1,2]}$ of $S O(3)$ group. In large external field limit, Hamiltonian (4) is reduced to

$$
H=B L_{z}+H_{0} .
$$

In this section, we begin with the above Hamiltonian to discuss the soliton excitations.

To describe the spin excitation, we introduce the boson excitation by the Holstein-Primakoff transformation 22],

$$
\begin{aligned}
L_{i}^{+} & =\hat{a}_{j}^{\dagger} \sqrt{2 S-\hat{a}_{j}^{\dagger} \hat{a}_{j}}, \\
L_{i}^{-} & =\sqrt{2 S-\hat{a}_{j}^{\dagger} \hat{a}_{j}} \hat{a}_{j}, \\
L_{i}^{z} & =\hat{a}_{j}^{\dagger} \hat{a}_{j}-S,
\end{aligned}
$$

where the annihilation operators $\hat{a}_{i}$ and the creation operators $\hat{a}_{j}^{\dagger}$ satisfy the boson commutation relation $\left[\hat{a}_{i}, \hat{a}_{j}^{\dagger}\right]=\delta_{i j}$. The number operator $\hat{n}_{i}=\hat{a}_{i}^{\dagger} \hat{a}_{i}$ characterizes the spin deviation from its maximum value $S$ of $L_{i}^{z}$. Usually, for a Heisenberg ferromagnetic system with inter-spin couplings $-J \mathbf{L}_{i} \cdot \mathbf{L}_{i+1}(J>0)$, the spontaneous symmetry breaking will happen along the direction of the external magnetic field (says along $z$-axis), which is subsequently assumed to approach zero. The ground state implies a ferromagnetic order with all spins along the $+z$ or $-z$ direction. Therefore the magnetization comes into being, which is defined as the non-vanishing average of $L^{z}=\sum_{i} L_{i}^{z}$. The governing equation for the nonlinear excitations on ground states can be obtained by the expanding $\sqrt{2 S-\hat{n}_{i}} \simeq \sqrt{2 S}\left(1-\hat{n}_{i} /(4 S)\right)$ to a series over the low excitation $\left\langle\hat{n}_{i}\right\rangle \ll 2 S$. We first expand (11a) and (11b), and keep the terms in the first order of $\left\langle\hat{n}_{i}\right\rangle$

$$
\begin{aligned}
& L_{i}^{+} \approx \sqrt{2 S} \hat{a}_{j}^{\dagger}\left(1-\frac{1}{4 S} \hat{a}_{j}^{\dagger} \hat{a}_{j}\right), \\
& L_{i}^{-} \approx \sqrt{2 S}\left(1-\frac{1}{4 S} \hat{a}_{j}^{\dagger} \hat{a}_{j}\right) \hat{a}_{j} .
\end{aligned}
$$

Consequently, beyond the spin wave approximation, the low energy effective Hamiltonian (10) is achieved as

$$
\begin{aligned}
H= & -c_{0} S \sum_{j}\left(\hat{a}_{j}^{\dagger} \hat{a}_{j+1}+h . c\right)+B \sum_{j} n_{j} \\
& +\frac{c_{0}}{4} \sum_{j}\left(\hat{a}_{j}^{\dagger} n_{j} \hat{a}_{j+1}+n_{j} \hat{a}_{j} \hat{a}_{j+1}^{\dagger}+h . c\right) \\
& -\left(c_{0}+c_{1}\right) \sum_{j} n_{j}\left(n_{j+1}-2 S\right),
\end{aligned}
$$

where $n_{j}=\hat{a}_{j}^{\dagger} \hat{a}_{j}, \quad c_{0}=J\left(1+\delta \sin ^{2} \theta / 2\right)$ and $c_{1}=$ $\delta J\left(3 \cos ^{2} \theta-1\right) / 2$.

As we should emphasize that, the external magnetic field has been assumed $B>0$ in the above discussions. If $B<0$, another form of Holstein-Primakoff transfor- mation should be taken

$$
\begin{aligned}
L_{i}^{+} & =\sqrt{2 S-\hat{a}_{j}^{\dagger} \hat{a}_{j}} \hat{a}_{j}, \\
L_{i}^{-} & =\hat{a}_{j}^{\dagger} \sqrt{2 S-\hat{a}_{j}^{\dagger} \hat{a}_{j}}, \\
L_{i}^{z} & =S-\hat{a}_{j}^{\dagger} \hat{a}_{j},
\end{aligned}
$$

but the main result of this paper is same. So without loss of generality, $B>0$ is assumed in the rest of this paper.

Hamiltonian (13) characterizes the low energy nonlinear property of ferromagnetic spin chain in an oblique magnetic field. The corresponding Heisenberg equation

$$
\begin{aligned}
i \hbar \frac{d \hat{a}_{j}}{d t}= & -c_{0} S\left(\hat{a}_{j+1}+\hat{a}_{j-1}\right)+\left(2 S\left(c_{0}+c_{1}\right)+B\right) \hat{a}_{j} \\
& +\frac{c_{0}}{4}\left(2 n_{j} \hat{a}_{j \pm 1}+n_{j \pm 1} \hat{a}_{j \pm 1}+\hat{a}_{j \pm 1}^{\dagger} \hat{a}_{j}^{2}\right) \\
& -\left(c_{0}+c_{1}\right) \hat{a}_{j} n_{j \pm 1}
\end{aligned}
$$

contains various nonlinear couplings. They lead to different nonlinear "phases", which are represented by various types of soliton excitations.

\section{CONTINUUM FIELD APPROACH FOR NONLINEAR COLLECTIVE EXCITATION}

In this section, we make use of the continuum field theory to study the nonlinear excitations in the ferromagnetic spin chain. To this end we consider the spin wave approach with some nonlinear corrections. First let us introduce the $p$-representation (or call the Glauber coherent state representation [23]) defined by the product of the multi-mode coherent states $|\alpha\rangle=\prod_{i}\left|\alpha_{i}\right\rangle$, where each component $\left|\alpha_{i}\right\rangle$ is the eigenstate of the annihilation operator $\hat{a}_{i}$, i.e., $\hat{a}_{i}\left|\alpha_{i}\right\rangle=\alpha_{i}\left|\alpha_{i}\right\rangle$, and $\alpha_{i}$ is the coherent amplitude. Since coherent states are normalized and overcompleted, the field operator sandwiched by $|\alpha\rangle$ can be represented only with their diagonal elements. Thus, we only need to consider the diagonal part of Eq. (15), which are enough to describe the nonlinear dynamics without any help of off-diagonal elements. The p-representation of non-linear equations (15) reads

$$
\begin{aligned}
i \hbar \frac{d \alpha_{j}}{d t}= & -c_{0} S\left(\alpha_{j \pm 1}-2 \alpha_{j}\right)+\left(2 S c_{1}+B\right) \alpha_{j} \\
& +\frac{c_{0}}{4}\left(2\left|\alpha_{j}\right|^{2} \alpha_{j \pm 1}+\left|\alpha_{j \pm 1}\right|^{2} \alpha_{j \pm 1}+\alpha_{j \pm 1}^{*} \alpha_{j} \alpha_{j}\right) \\
& -\left(c_{0}+c_{1}\right) \alpha_{j}\left|\alpha_{j \pm 1}\right|^{2} .
\end{aligned}
$$

From then on, the spin dynamics is expressed in terms of the $c$-number equation in Glauber's coherent-state representation. However Eq. (16) is difficult to solve due to its nonlinearity and discreteness. Since we are looking for excitations with a length scale much larger than the lattice constant, we take the long-wave approximation, that is, the continuum field theory approach will be employed in the large $N$ limit. By assuming that the 
coherent amplitude is continuum in space, the discrete variables $\alpha_{i}(t)$ can be replaced by a mean field $\varphi(z, t)$, i.e. $\alpha_{i}(t) \rightarrow \varphi(z, t)$. Correspondingly, the difference become the following differential

$$
\alpha_{i \pm 1}-\alpha_{i} \rightarrow \pm \frac{\partial}{\partial z} \varphi+\frac{1}{2} \frac{\partial^{2}}{\partial z^{2}} \varphi+\cdots,
$$

where the lattice constant is assumed to be unity. By keeping the derivation terms to the second order $\partial^{2} / \partial z^{2}$, the system of equations (16) become a field equation with self-coupling terms

$$
i \hbar \frac{d}{d t} \varphi+c_{0} S \frac{\partial^{2}}{\partial x^{2}} \varphi+2 c_{1} \varphi|\varphi|^{2}=V \varphi,
$$

which is a typical NLS equation in a constant potential $V=2 S c_{1}+B$.

To separate the rapid oscillation in the inhomogeneous nonlinear equation (18), we rewrite $\varphi(z, t)$ as

$$
\varphi(z, t)=e^{-i \chi t} \phi(\xi, t)
$$

where $\xi=x \sqrt{\hbar /\left(c_{0} S\right)}, \chi=\left(2 S c_{1}+B\right) / \hbar$, and $\phi(\xi, t)$ is the slow varying envelope. Then the standard form of the NLS equation is obtained

$$
i \phi_{t}+\phi_{\xi \xi}+2 \frac{c_{1}}{\hbar}|\phi|^{2} \phi=0
$$

which has the soliton solution. Here, the strength of nonlinear term

$$
c_{1}=\frac{\delta}{2} J\left(3 \cos ^{2} \theta-1\right)
$$

is determined by spin-spin interaction parameter $J$ and the angle $\theta$ of the oblique magnetic field. We notice that when $\theta=\theta_{0}=\arccos \sqrt{1 / 3}$, the nonlinear term in the equation of the motion disappears, therefore Eq.(20) becomes a standard wave equation, which gives a complete set of plane waves solution. In this case, the collective excitations are spin waves. $\theta_{0}$ is named as the magic angle. It shows that the magic angle changes the anisotropic system to the isotropic one.

\section{NONLINEAR TIME EVOLUTION OF LOCALIZED MAGNETIZATION}

For this ferromagnetic spin chain, the interaction parameter $J$ is positive. Thus the sign of coefficient $c_{1}$ is determined by the angle of the oblique magnetic field $\theta$. And nonlinear property of this system is strongly related with the sign of the nonlinear term. With these different physically accessible parameters, the nonlinear equation (20) can possess the bright-soliton and the dark-soliton solution. Next, we use the inverse scattering method [24, 25] to obtain the different solitons of this system.

When $c_{1}>0$, that is $\delta\left(3 \cos ^{2} \theta-1\right)>0$, the single bright soliton solution of Eq.(18) is obtained as

$$
\varphi(x, t)=A e^{i(\gamma x-\omega t)} \operatorname{sech}\left[A \sqrt{\frac{c_{1}}{c_{0} S}}\left(x-x_{0}-v t\right)\right],
$$

with $v=v_{1} \sqrt{c_{0} S / \hbar}, \gamma=\hbar v / 2 c_{0} S$ and

$$
\omega=\frac{c_{1}}{\hbar}\left(2 S-A^{2}\right)+\frac{B}{\hbar}+\frac{\hbar v^{2}}{4 c_{0} S}
$$

where $v_{1}$ is an integral constant. The coefficient $v=$ $v_{1} \sqrt{c_{0} S / \hbar}$ describes the velocity of bright soliton traveling to the right. The positive coefficient $A$ characterizes the size of the bright soliton. The coefficient $x_{0}$ denotes the initial position of the bright soliton. Parameters $v_{1}$, $A$ and $x_{0}$ are determined by the initial state. The bright soliton solution (22) depicts a wave packet traveling in the continuous background.

When $c_{1}<0$, that is $\delta\left(3 \cos ^{2} \theta-1\right)<0$, we can get a single dark-soliton solution of Eq.(18)

$$
\varphi(x, t)=A^{\prime} e^{i\left(\gamma^{\prime} x-\omega^{\prime} t\right)} \tanh \left[A^{\prime} \sqrt{\frac{-c_{1}}{c_{0} S}}\left(x-x_{0}^{\prime}-v^{\prime} t\right)\right]
$$

with $v^{\prime}=v_{1}^{\prime} \sqrt{c_{0} S / \hbar}, \gamma^{\prime}=\frac{\hbar v^{\prime}}{2 c_{0} S}$, and

$$
\omega^{\prime}=2 \frac{c_{1}}{\hbar}\left(S-A^{\prime 2}\right)+\frac{B}{\hbar}+\frac{\hbar v^{\prime 2}}{4 c_{0} S}
$$

where $v_{1}^{\prime}$ is an integral constant. $v^{\prime}=v_{1}^{\prime} \sqrt{c_{0} S / \hbar}$ characterizes the velocity of dark soliton traveling to the right. The positive coefficient $A^{\prime}$ characterizes the size of the dark soliton. The coefficient $x_{0}^{\prime}$ denotes the initial position of the dark soliton. Parameters $v^{\prime}, A^{\prime}$ and $x_{0}^{\prime}$ are decided by the initial state. The dark soliton solution describes a localized dip in the continuous background. Fig. 11numerically illustrates the bright and the dark soliton obtained from Eq. (22) and (24) respectively in comparison with the results with many extra nonlinear terms in the following discussion.

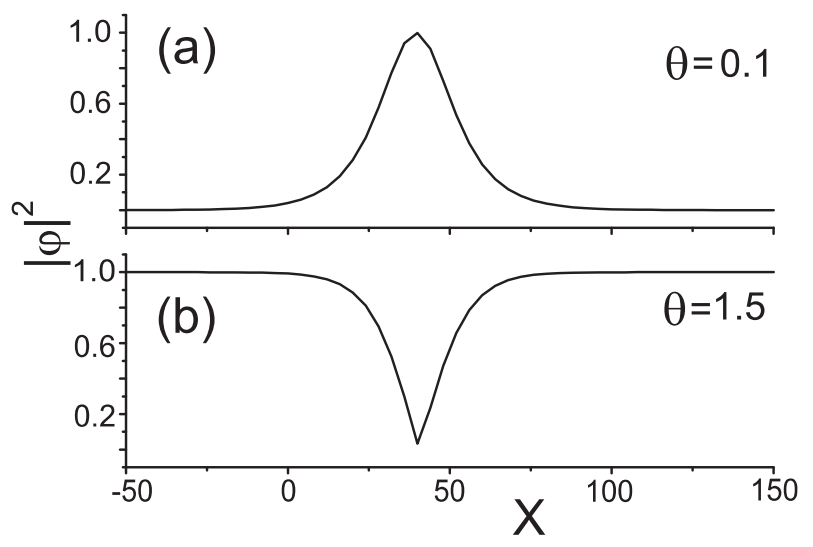

FIG. 1: Bright soltion (a) obtained from Eq. (22) for $\theta=0.1$. Dark soltion (b) obtained from Eq. (24) for $\theta=1.5$. Other parameters are taken as follows $S=10, B=100, J=1, \delta=$ $0.1, x_{0}=x_{0}^{\prime}=0, A=A^{\prime}=1, v_{1}=v_{1}^{\prime}=5$ and $t=3$.

Furthermore, to test the rationality for neglecting the terms $H_{ \pm 1}$ and $H_{ \pm 2}$, we deal with Hamiltonian (4) by the approach presented above. The Holstein-Primakoff transformation (11) is first employed to write Hamiltonian (4) in terms of bosonic operators 


$$
\begin{aligned}
H= & -c_{0} S \sum_{j}\left(\hat{a}_{j}^{\dagger} \hat{a}_{j+1}+\hat{a}_{j} \hat{a}_{j+1}^{\dagger}\right)+B \sum_{j} n_{j}-\left(c_{0}+c_{1}\right) \sum_{j} n_{j}\left(n_{j+1}-2 S\right) \\
& +\frac{c_{0}}{4} \sum_{j}\left(\hat{a}_{j}^{\dagger} n_{j} \hat{a}_{j+1}+\hat{a}_{j}^{\dagger} n_{j+1} \hat{a}_{j+1}+\text { h.c. }\right)+c_{2} \sqrt{2 S} \sum_{j}\left(\hat{a}_{j}^{\dagger} n_{j \pm 1}+\frac{1}{2} \hat{a}_{j}^{\dagger} n_{j}-2 S \hat{a}_{j}^{\dagger}+\text { h.c. }\right) \\
& -2 c_{3} S \sum_{j}\left(-\frac{1}{4 S}\left(\hat{a}_{j}^{\dagger} n_{j} \hat{a}_{j+1}^{\dagger}+\hat{a}_{j}^{\dagger} \hat{a}_{j+1}^{\dagger} n_{j+1}\right)+\hat{a}_{j}^{\dagger} \hat{a}_{j+1}^{\dagger}+\text { h.c. }\right),
\end{aligned}
$$

where $c_{0}=J\left(1+\delta \sin ^{2} \theta / 2\right), c_{1}=J \delta\left(3 \cos ^{2} \theta-1\right) / 2$, $c_{2}=J \delta \sin 2 \theta / 4$ and $c_{3}=J \delta \sin ^{2} \theta / 4$. Hamiltonian (26) characterizes the low energy nonlinear property of ferro- magnetic spin chain in an oblique magnetic field. The corresponding Heisenberg equation

$$
\begin{aligned}
i \hbar \frac{d \hat{a}_{j}}{d t}= & -c_{0} S \hat{a}_{j \pm 1}+\left(B+2 S\left(c_{0}+c_{1}\right)\right) \hat{a}_{j}-\left(c_{0}+c_{1}\right) \hat{a}_{j} n_{j \pm 1}+\frac{c_{0}}{4}\left(2 n_{j} \hat{a}_{j \pm 1}+n_{j \pm 1} \hat{a}_{j \pm 1}+\hat{a}_{j \pm 1}^{\dagger} \hat{a}_{j} \hat{a}_{j}\right) \\
& +c_{2} \sqrt{2 S}\left(n_{j \pm 1}+\hat{a}_{j \pm 1}^{\dagger} \hat{a}_{j}+\hat{a}_{j \pm 1} \hat{a}_{j}-2 S+n_{j}+\frac{1}{2} \hat{a}_{j} \hat{a}_{j}\right) \\
& -c_{3} 2 S\left(\hat{a}_{j \pm 1}^{\dagger}-\frac{1}{2 S} n_{j} \hat{a}_{j \pm 1}^{\dagger}-\frac{1}{4 S} \hat{a}_{j \pm 1}^{\dagger} n_{j \pm 1}-\frac{1}{4 S} \hat{a}_{j} \hat{a}_{j} \hat{a}_{j \pm 1}\right)
\end{aligned}
$$

contains rich nonlinear couplings and thus can predict various nonlinear phenomena.

Further we use the continuum field theory approach, that is, expressing the spin dynamics according to its corresponding continuous c-number equation in Glauber's coherent-state representation. Then a similar field equation is obtained as

$$
\begin{aligned}
& i \hbar \frac{d}{d t} \varphi+c_{0} S \frac{\partial^{2}}{\partial x^{2}} \varphi+2 c_{1} \varphi|\varphi|^{2} \\
= & \left(2 S c_{1}+B\right) \varphi+2 c_{2} \sqrt{2 S}\left(\frac{5}{2}|\varphi|^{2}+\frac{5}{4} \varphi^{2}-S\right) \\
& -c_{3}\left(4 S \varphi^{*}-3 \varphi^{*}|\varphi|^{2}-\varphi^{3}\right) .
\end{aligned}
$$

Obviously, the effects on $H_{ \pm 1}$ and $H_{ \pm 2}$ is kept in the above derivation. Eq.(28) is similar to the NLS equation, but more nonlinear terms are involved, therefore the analytic solution can not be got.

To display the effects of extra nonlinear terms, we numerically investigate the time evolution of dark and bright solitons under the action of differential equation (28) in Fig. 2 and Fig. 3 , Figures 2 and 3 show that a bright and a dark solition can be excited when $\theta$ takes value on the left and the right side of the magic angle respectively. Comparing the numerical results shown in Fig. 2] and 3, it is found that the bright soliton is more stable than the dark soliton solution. This is because the long time evolution of the dark soliton in Fig. 3 become a profile with spatial oscillation. While the bright soli- ton will almost keep their shapes, the dark soliton will disappear after a long enough evolution. Thus the bright soliton is more easily detectable in practice. From Fig. 2 , we found that the closer to the magic angle the tilted angle is, the wider the soliton becomes.

In Fig. 4. we plot the time evolution for the stable bright soliton with respect to different values of the dimensionless parameter $\lambda=B / J$. It can be found that the extra nonlinear terms has no effect on solitons when $\lambda=100$. Such numerical analysis confirms that the soliton-like wave can be excited under an appropriate regime from $\lambda=10$ to 1000 approximately. Therefore in this regime, it is reasonable to discard the terms $H_{ \pm 1}$ and $H_{ \pm 2}$ in Eq. (4). When parameters $\lambda$ goes to infinite (see the line of $\lambda=5000$ in Fig (4), the interaction term $H_{0}$ can be completely ignored, then Hamiltonian of the system approximately described by $B L_{z}$. In this situation, the system is completely polarized along the direction of the magnetic field and form a background with symmetry breaking which is displayed by the dot line in Fig. 4. The numerical result that a very sharp peak is localized around $x=0$ justifies our observation from the physical intuition.

There are some experimental data on quasi-onedimensional ferromagnetic chains [27, 28, 29, 30, 31], which may be used to test our predictions in an indirect way. For the spin chain made of the material $\mathrm{Ca}_{3} \mathrm{Co}_{2-x} \mathrm{Fe}_{x} \mathrm{O}_{6}(\mathrm{x}=0,0.1,0.2)$ (Ref. [27]), the exchange constant $J$ decreases from 4.39 to $8.13 K$ and the mag- 


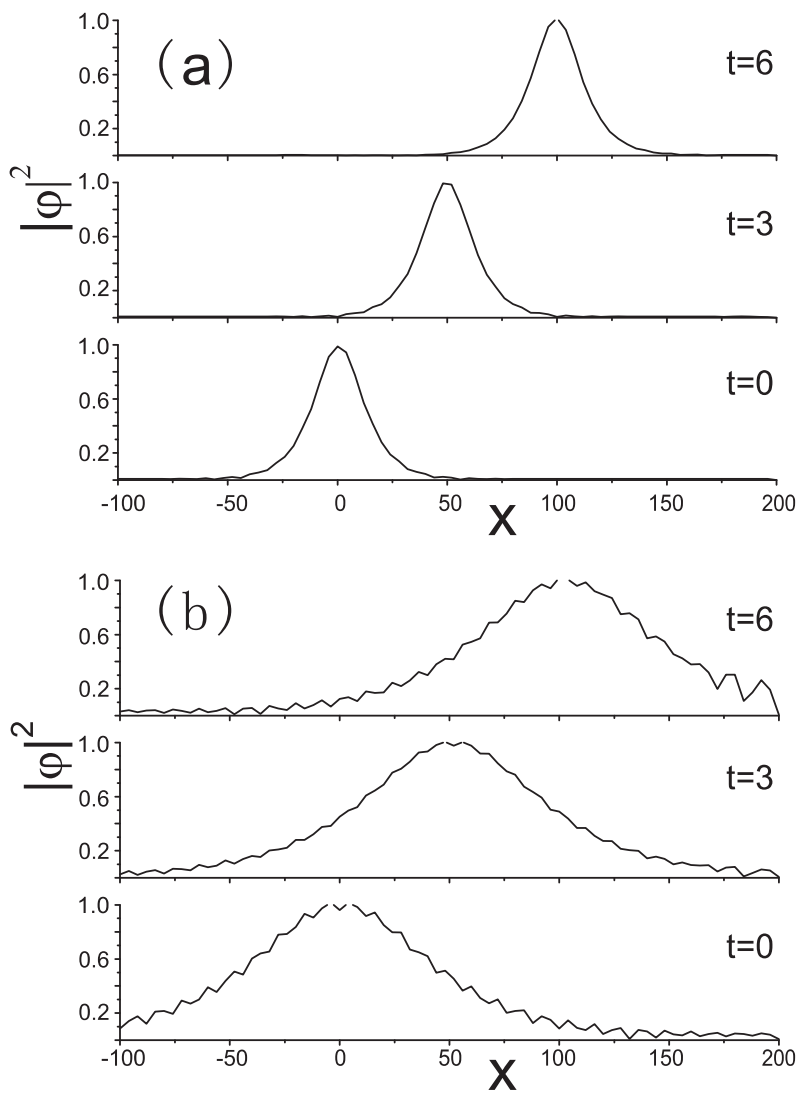

FIG. 2: Numerical time evolution of a bright soliton under the act of Eq. (28) for $\theta=0.1$ (a) and $\theta=0.9$ (b). Other parameters are taken as follows $S=10, B=100, J=1$ and $\delta=0.1$. It indicates that a bright soliton is excited when $\theta<\theta_{0}$.

netic field strength $B<10 T(\sim 33.6 K)$. The solitaryexcitation is possible since the dimensionless parameter $\lambda=B / J \approx 10$. However, for the three ferromagnetic chains [28, 29, 30, 31], which made of the following material: (1) $\left[\left(\mathrm{CH}_{3}\right)_{3} \mathrm{NH}\right] \mathrm{FeCl}_{3} \cdot 2 \mathrm{H}_{2} \mathrm{O}$ (Ref. 28,29$]$ ), where $J \sim 17.4 K, B<16 T(\sim 21.5 K) ;(2) \mathrm{CoCl}_{2} \cdot 2 \mathrm{D}_{2} \mathrm{O}$ (Ref. [30]), where $J \sim 2.475 K$ and $B<6 T(\sim 12.1 K)$; (3) $\mathrm{CoCl}_{2} \cdot \mathrm{H}_{2} \mathrm{O}$ (Ref. [31]), $J \sim 18.3 \mathrm{~K}$ and $B<6 T$ $(\sim 12.1 K)$, the bright soliton may be excited, but noises exist as shown in Fig. 4, with the dimensionless parameter $\lambda=B / J \approx 1$.

\section{REMARKS AND CONCLUSION}

Before concluding this paper, let us discuss the physical meaning of the solitary wave of magnetic excitation in a $N$-site $X X Z$ quantum spin chain from the point view of quantum information processing. We first note that the multi-mode coherent states $|\alpha\rangle=\prod_{j}\left|\alpha_{j}\right\rangle$ represent an inhomogeneous collective excitations distributed around the spin chain. This collective excitation has a
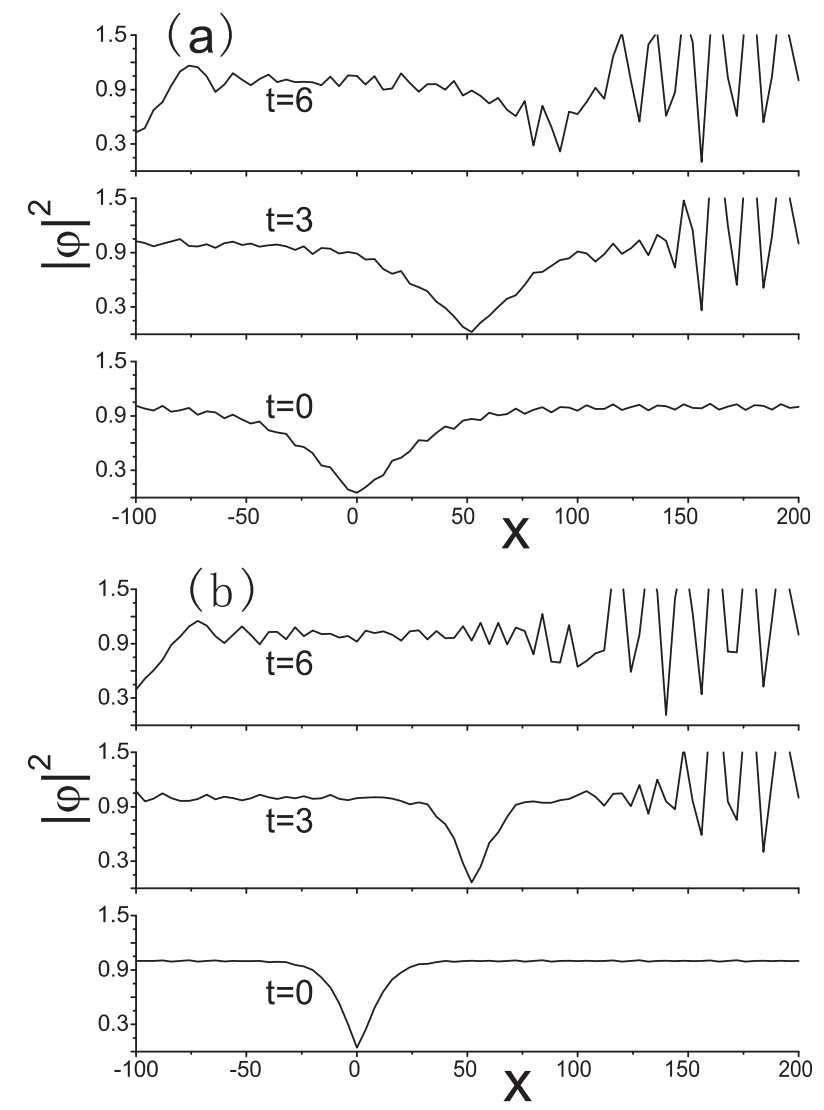

FIG. 3: Numerical time evolution of a dark soliton by the influence of extra nonlinear terms in Eq. (28) for $\theta=1.0$ (a) and $\theta=1.5$ (b). Other parameters are taken as follows $S=10, B=100, J=1$ and $\delta=0.1$. It indicates that a dark soliton is excited when $\theta$ takes value on the right side of the magic angle.

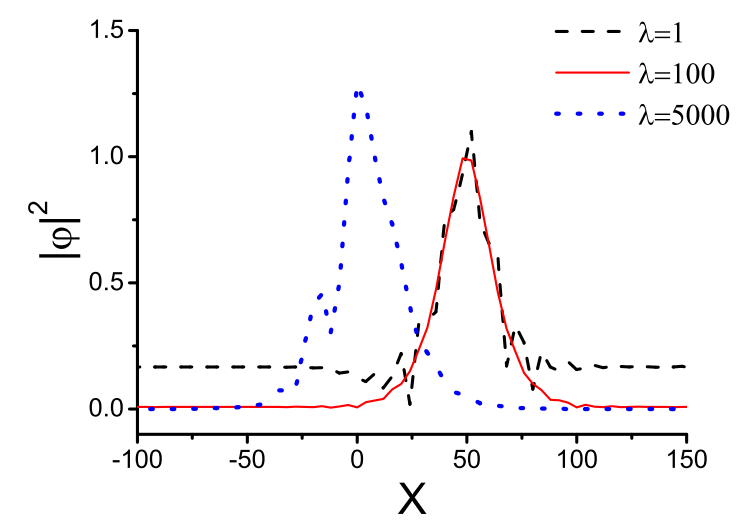

FIG. 4: (Color online) the time evolution of a bright soliton under the effluence of extra nonlinear terms in Eq. (28) with respect to different values of the dimensionless parameter $\lambda=$ $B / J$ at $\theta=0.1, S=10, \delta=0.1, t=3$. 
spin representation by

$$
\hat{a}_{j}^{\dagger}=L_{i}^{+} \frac{1}{\sqrt{S-L_{i}^{z}}} \equiv Q L_{i}^{+}
$$

with $Q=1 / \sqrt{S-L_{i}^{z}+1}$ and $|0\rangle_{j}=|S,-S\rangle_{j}$ being the lowest eigenstate of the on-site spin $\mathbf{L}_{j}$. We also notice that the coherent state denotes a superposition of various spin states $\left|S, m_{s}\right\rangle_{j}$, i.e.,

$$
\left|\alpha_{i}\right\rangle=\sum C_{n}\left(\alpha_{i}\right)\left(Q L_{i}^{+}\right)^{n}|S,-S\rangle_{j}=\sum B_{n}\left(\alpha_{i}\right)|S, n-S\rangle_{j}
$$

where $C_{n}\left(\alpha_{i}\right)=\exp \left(-\left|\alpha_{j}\right|^{2} / 2\right) \alpha_{j}^{n_{j}} /\left(n_{j} !\right)$ and $B_{n}\left(\alpha_{i}\right)$ can be obtained from $C_{n}\left(\alpha_{i}\right)$ directly. During the propagation of soliton, the narrow traveling wave packet does not spread, therefore it behaves like a "flying qudit" (a $d=2 S$ level system).

When $S=1 / 2$, the qubit is localized at the $j$ th site with a superposition state

$$
\left|\alpha_{j}\right\rangle \sim\left|\frac{1}{2},-\frac{1}{2}\right\rangle+\frac{\alpha_{j}}{2}\left|\frac{1}{2}, \frac{1}{2}\right\rangle
$$

which can be used to encode quantum information as usual. During the propagation, the wave function nearly keeps its spatial shape all the time. From an mathematical point of view, the spatially non-spreading properties of the carrying excitation wave is very crucial for quantum state transfer from one location to another with high fidelity. It seems the bosonic excitations obey the bosonic commutation relations only in the large $S$ limit, but the spin wave approach can still work well for $S=1 / 2$ in the condensed matter physics. In this sense we can suppose our above arguments available.

In summary, we have studied solitary magnetic excitation in a $N$-site $X X Z$ quantum spin chain as well as how to use an oblique magnetic field to create different types of solitons. Through a mean field approximation beyond the usual spin wave approach, we obtain the quasi-classical motion equations for nonlinear evolution of the Heisenberg spin system. We show that the switch between the bright and the dark soliton is controlled by the angle of the magnetic field, whose tangent is defined by the ratio between the $\mathrm{z}$ component and the $\mathrm{x}$ component of the magnetic field. And at the magic angle, the system go to the isotropic Heisenberg model, hence, only an ideal spin wave is stimulated. We also remark the possibility for solitons to play the role of "flying qudit" based on the well-known results in Ref. 26] that a no-spreading wave-packet behaves like a "flying qubit".

This work is supported by the NSFC with grant Nos. 90203018, 10474104, 60433050, 10325523, 10347128,10075018 and 10704023 , the NFRPC with Nos. 2001CB309310 and 2005CB724508, and the Scientific Research Fund of Hunan Provincial Education Department of China (Grant No. 07C579).
[1] S. Bose, Phys. Rev. Lett. 91, 207901 (2003).

[2] Y. Li, T. Shi, B. Chen, Z. Song, and C.-P. Sun, Phys. Rev. A 71, 022301 (2005).

[3] T. Shi, Y. Li, Z. Song, and C.-P. Sun, Phys. Rev. A 71, 032309 (2005).

[4] M. Christandl, N. Datta, A. Ekert, and A. J. Landahl, Phys. Rev. Lett. 92, 187902 (2004).

[5] L. D. Faddeev and L. A. Takhtajan, Hamiltonian Methods in the Theory of Solitons (Springer, Berlin, 1987).

[6] Y. S. Kivshar and B. A. Malomed, Rev. Mod. Phys. 61, 763 (1989); 63, 211 (1991).

[7] V. G. Bar'yakhtar, M. V. Chetkin, B. A. Ivanov, and S. N. Gadetskii, Dynamics of Topological Magnetic Solitons (Springer, Berlin, 1994).

[8] N. N. Huang, Z. Y. Chen, and Z. Z. Liu, Phys. Rev. Lett. 75, 1395 (1995).

[9] M. M. Fogler, Phys. Rev. Lett. 88, 186402 (2002).

[10] C. P. Slichter, Principles of Magnetic Resonance (Springer, Berlin, 1996).

[11] A. M. Kosevich, B. A. Ivanoy, and A. S. Kovalev, Phys. Rep. 194, 117 (1990).

[12] J. Tjon and J. Wright, Phys. Rev. B 15, 3470 (1977).

[13] H. C. Fogedby, J. Phys. A 13, 1467 (1980).

[14] D. I. Pushkarov and Kh. I. Pushkarov, Phys. Lett. A 61, 339 (1977).

[15] R. Balakrishnan and A. R. Bishop, Phys. Rev. Lett. 55, 537 (1985).
[16] R. Ferrer, Phys. Rev. B 40, 11007 (1989).

[17] M. J. Skrinjar, D. V. Kapor and S. D. Stojanovic, J. Phys. Condens. Matter 1, 725 (1989).

[18] Z.-P. Shi, G. Huang and R. Tao, Phys. Rev. B 42, 747 (1990).

[19] M. Daniel, L. Kavitha and R. Amuda, Phys. Rev. B 59, 13774 (1999).

[20] E. Lieb and D. Mattis, J. Math. Phys. 3, 749 (1962).

[21] I. Affleck and E. Lieb, Lett. Math. Phys. 12, 57 (1986).

[22] T. Holstein and H. Primakoff, Phys. Rev. 58, 1098 (1940).

[23] R. J. Glauber, Phys. Rev. 131, 2766 (1963).

[24] R. K. Dodd, J. C. Eilbeck, J. D. Gibbon, and H. C. Morris, Solitons and Nonlinear Wave Equations (Academic, London, 1982).

[25] M. Ablowitz and H. Segur, Solitons and the Inverse Scattering Transform (SIAM, Philadelphia, 1981).

[26] S. Yang, Z. Song and C. P. Sun, Phys. Rev. A 73, 022317 (2006).

[27] A. Jain, Sher Singh, and S. M. Yusuf, Phys. Rev. B 74, 174419 (2006).

[28] R. S. Rubins, A. Sohn, T. D. Black, and John E. Drumheller, Phys. Rev. B 61, 11259 (2000).

[29] R. E. Greeney, C. P. Landee, J. H. Zhang, and W. M. Reiff, Phys. Rev. B 39, 12200 (1989).

[30] W. Montfrooij, G. E. Granroth, D. G. Mandrus, and S. E. Nagler, Phys. Rev. B 64, 134426 (2001). 
[31] J. B. Torrance, Jr. and M. Tinkham Phys. Rev. 187, 595 (1969). 